\title{
Who wants to be a self-driving car?
}

\author{
A mixed-reality data-trust exercise
}

\section{Keywords: VR, mixed reality, empathy prototype, data trust, autonomous vehicles}

\section{Self-driving cars and autonomous transportation systems} are projected to create radical societal changes, yet public understanding and trust of self-driving cars and autonomous systems is limited. The authors present a new mixed-reality experience designed to provide its users with insights into the ways that self-driving cars operate. A single-person vehicle equipped with sensors provides its users with data driven visual feedback in a virtual reality headset to navigate in physical space. The authors explore how immersive experiences might provide 'conceptual affordances' that lower the entry barrier for diverse audiences to discuss complex topics.

\section{Background}

Self-driving cars and autonomous transportation systems are projected to create potentially radical societal changes (Evans 2017). As the technological ecosystem around self-driving cars makes significant progress and the legal infrastructure to govern autonomous mobility systems continues to develop, research on issues related to trust, public perception, and user experiences/interactions in a future with self-driving cars begins to surface
(Deloitte 2017). Studies show that, in general, people are currently distrustful of and uncomfortable with the idea of being transported by self-driving cars, especially fullyautonomous ones (e.g., without a steering wheel) (KBB 2016). In addition, studies show that levels of trust and acceptance of self-driving cars vary according to factors such as country of origin, gender, and age (Howard \& Dai 2013), though overall, there is a general lack of knowledge about autonomous vehicles by the public (КвB 2016).

At the same time, there is a growing body of work drawing attention to how machines 'see the world'. The research explores the artifacts of a robot readable worldfrom the signals produced from sensors and computers and the aesthetic qualities of those features such as noise and glitch to the implications of how and what machines see (Arnall 2012). These explorations are best described by James Bridle's and by Matt Jones's definitions of "the new aesthetic" or "the sensor vernacular", which speak not only to the aesthetics of machine vision, but also how they influence or change human behavior (Bridle 2019, Jones 2011). These explorations are not only happening in a media art context but have become a critical research question in understanding the decision making process of artificial intelligence (Knight 2017, Sütfeld et al. 2017). What these works indicate is a growing need for rendering computational systems more readable, visible, and actionable. 
By revealing how machines see, is it possible to create new entry points for a wider audience to discuss the varying roles that technology have in society? And if so, how might an immersive, mixed-reality experience be positioned, presented, and facilitated to engage diverse audiences with a complex topic such as the layers of discussion-e.g., political, legal, cultural, and technological-surrounding self-driving cars?

Researchers and designers have been employing speculative design methods to build scenarios that explore public perception of and interaction with self-driving cars. These speculations normalize or assume certain key conditions that help to make speculative scenarios more real (Dunne \& Raby 2013). For example, academic and applied researchers are exploring both the optics and the interaction of people with self-driving cars. Studies such as those by Guess (2017) and Aouf (2018) point out that the future of self-driving cars relies not only on how cars see people, but how people see self-driving cars. Projects such as IDEO's (2019) Automobility Project envision a more probable and commercial future while Jonathan Keats' collaboration with Nissan envisions alternatives which may presume a passive engagement while riding a self-driving car. Furthermore, projects such as MIT's moral machine (Awad et al. 2018) explore the trolley problem for self-driving cars through a game-based interactive survey.

This project seeks to add a set of reference points to the growing literature on the social, cultural, and technological changes that society is facing due to the integration of self-driving cars. The authors posit that through explorations such as their immersive experience, designers and researchers are contributing to a collective consciousness about the first, second, and perhaps even third order consequences that widespread autonomous vehicles, their software and their embedded politics will have for the world.

The goal of this case study is to present the authors' experience developing and operationalizing their project titled 'Who Wants to Be a Self-driving Car?'. This paper briefly explores the authors' takeaways engaging a diverse and international audience with their unconventional approach to user research and in promoting technological literacy.

\section{Problem}

The motivation behind this work was to create an accessible entry point into the topic of self-driving cars, allowing people to discuss, ask questions, and learn more about self-driving cars in a facilitated context. At the time of project development, there was a growth in literature and media interest around self-driving cars and the technologies and conditions that dictate their performance. The authors identified this as an opportunity to propose a new entry point for people to communicate how self-driving cars see through and make decisions based on data. The authors posit that given the potential impact that self-driving cars can have in shaping the future, there must be more and varied approaches to engaging diverse and international audiences with the topic.

\section{Methodology \& solution}

\subsection{Materials and production}

The aim of this immersive prototype was to build a machine which can replace human senses with the sensors that a self-driving car might use. Our unconventional driving machine, 'the vehicle', is essentially a steel-frame buggy with in-wheel, electric motors and hydraulic braking, and physical steering. Drivers lay head first on the vehicle, which is the positioning used to enhance the feeling of immersion (and vulnerability) created during the experience (Figure 1).

The VR experience is created using data collected by the sensors outfitted on the vehicle. The main view 


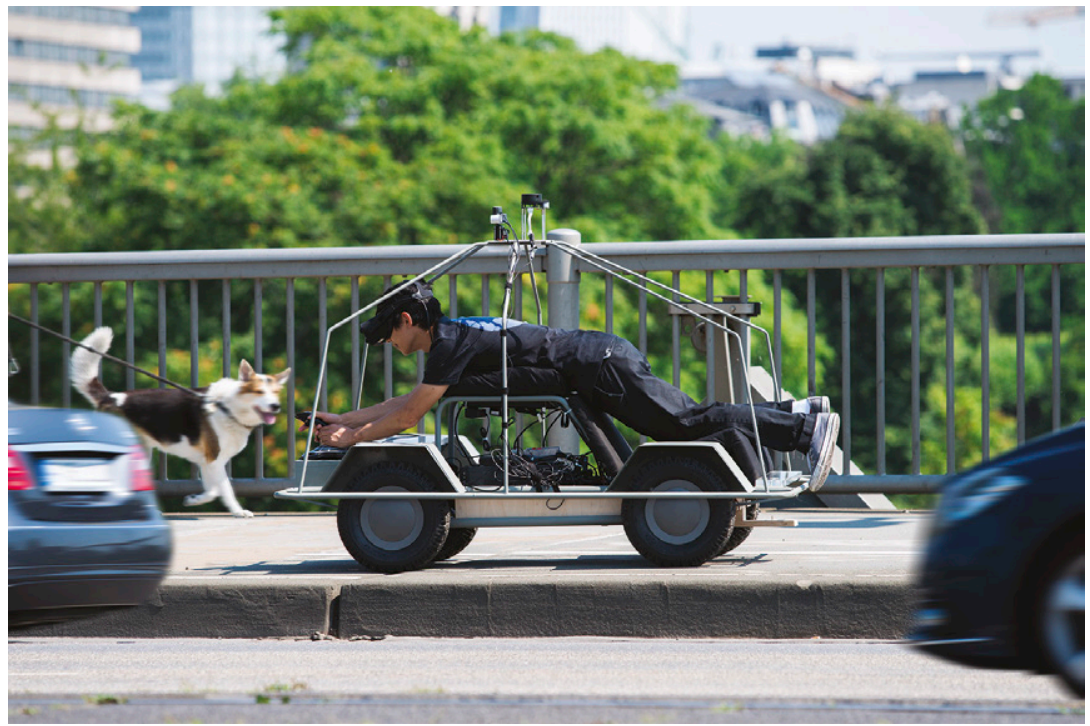

Figure 1. "Who Wants to be a Self-Driving Car" vehicle on a test drive (image by Raphael Reimann). is a presentation of data from a $3 \mathrm{D}$ depth camera that uses stereoscopic imaging to map the landscape in real time. The 3D mapping of the vehicle's vicinity is supplemented with visual object detection using the yoLO (Redmon et al. 2016) image recognition library, which adds another visible meta-data layer, further supporting the driver. Furthermore, a light detection and ranging (LiDAR) sensor adds an extra layer of distance sensing.

These visual components are pulled together into the VR headset using VvVv and the Unity 3D game engine to provide the drivers with data that they must interpret in order to navigate the driving machine through space. This essentially replaces the control unit of an autonomous vehicle with a person.

\subsection{VR view \& driving experience}

The data presented in the VR view (Figure 2) are a combination of the 3D depth data, the LiDAR points, and the detected and classified objects. The goal was to create a data visualization that could offer just enough actionable information for users to navigate with, while also communicating about the different ways in which machines process and understand data.

The authors abstract the 3D depth image to contour lines as a reference to the types of 'data gaps' that are present in $3 \mathrm{D}$ point cloud data produced by the iconic multi-channel LiDAR sensors that typically sit on top of self-driving cars. The abstraction helps us to discuss aspects of self-driving sensor technology such as data resolution, precision and accuracy, and how these affect how and what self-driving cars can see, while still being able to maneuver the vehicle.

The results of real-time object detection and classification are displayed in the VR view. The detection results are represented in different colors (e.g., blue for cars, green for people, grey for other elements) as pulsing lines radiating out from the driver to the detected object 


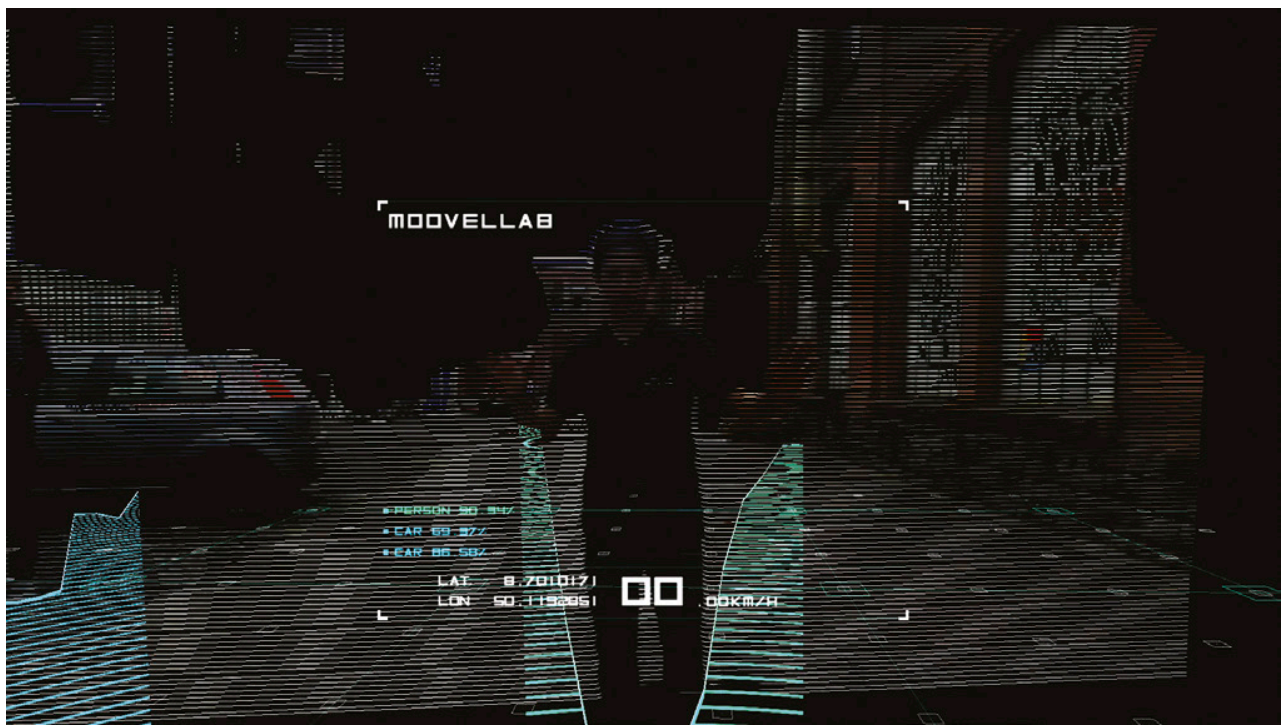

Figure 2. $2 \mathrm{D}$ export of VR view. Image by moovel lab. to create a sense of distance between the driver and the detected objects in the view.

\section{Results \& conclusions}

\subsection{Discussion and facilitation}

The project takes a user-centered approach to gathering feedback about the topic of self-driving technology. We do this by building empathy for machines and data-driven processes using mixed reality. From this perspective, the project creates an accessible entry point into the expansive topic of self-driving technology and broader trusting data, ranging from aspects of technology, business, law, ethics, privacy, and design.

The people who engage with the project therefore draw from their own experiences and/or are prompted to ask questions about the topic of self-driving cars. After being briefly on-boarded, participants are prompted to drive the vehicle around a given venue (Figure 3). The experience relies heavily on the facilitation of the creators of the project and their ability to engage with the participants. The project offers a medium for reflection on reframing and for learning about how the self-driving experience might occur in the future.

\subsection{Questioning one's senses}

This project was inspired by the question 'how do self-driving cars see the world?' From a technical point of view, the computers in self-driving cars receive complex matrices of numbers streaming in from the sensors that envelope them. The way self-driving cars constantly process 'what is seen' and the way they can statistically calculate the uncertainty of their decision-making are therefore fundamentally different from the subconscious 'data processing' and decision-making processes of the human mind. 


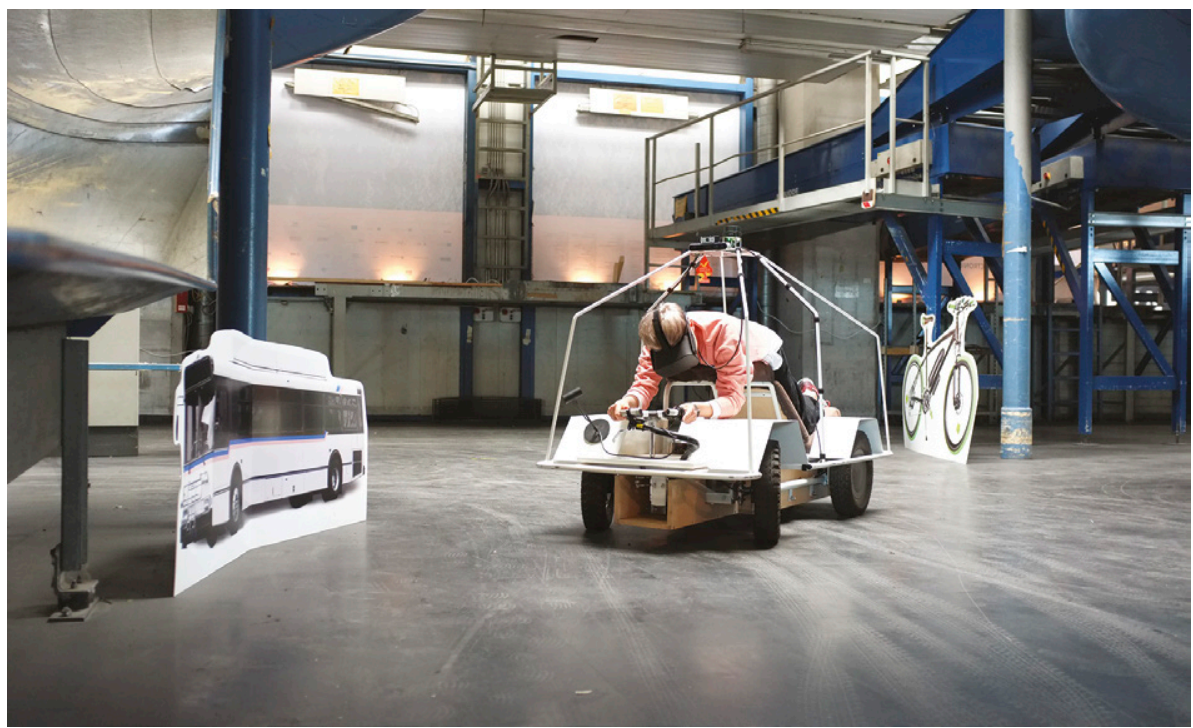

Figure 3. Exhibition setup with dummy traffic participants. Image by moovel lab.
During our research, we wanted to find out what it is that self-driving cars see (or do not see) that makes cars 'doubt' their ability to navigate with confidence. We also wanted to find out how we could create an experience that prompts people to question the amount and the quality of the data that are available to them. The empathy component of this data-trust exercise can be seen when people drive a vehicle equipped with only 'enough' sensors and data to navigate a prescribed area.

The purpose of this prototype is not to simulate 'how cars actually see the world', but rather to simulate the feeling of processing data in order to navigate. The visualizations in the VR headset are therefore radically simplified abstractions of data sources that, while incomprehensible to humans, allow self-driving cars to drive and make decisions about the environment. We argue that a fundamental characteristic of the technologies that support self-driving cars is that every decision is calculated as opposed to intuited.
To help people become aware of all the challenges that driving a car based on data presents is an ambitious and challenging task. Nevertheless, our project presents an opportunity for people to consider the implications of not only seeing the world through sensors but also being able to drive based on data alone.

\subsection{Future directions}

'Who Wants to be a Self-Driving Car?' is a tool to explore the technology behind self-driving cars from a human perspective. For most, the project is an entry point into a conversation about self-driving cars and their possible impacts on society. For some, however, the project prompts deeper questions about the new ways in which artificial intelligence, sensors, and mobility are connected.

This project can be seen as a proposal for developing deeper relationships between visualization and speculative design. One role of speculative design is to unpack 
uncertainty and create tangible expressions of models of the future. Speculative designs can create deeper expressions of the types of uncertainties and model projections communicated in or inspired by visualizations. While both speculative designs and visualizations offer opportunities to look into what is plausible, possible and probable, speculative designs also create opportunities to poke at what might be preferable (Dunne \& Raby 2013). Speculative design is therefore much like a map in that the scenarios-if done well and with rigor-can prompt self-reflection. For the authors, their work offers the possibility of exploring the deeper implications of what is 'in a data point', as well as of investigating how society may deal with these implications.

The authors believe that continued engagement with new interfaces and experiences which aim to demystify or unpack the complexity of intersecting and interacting technologies is a worthwhile endeavor, especially engagement with those experiences that invite diverse collaborations and interdisciplinary research.

\section{Submission date: 3 February, 2019 \\ Accepted date: 11 June, 2019}

\section{Acknowledgements}

Special thanks to the team at MESO Digital Interiors (Sebastian Oschatz, Theron Burger, Nikos Mechanezidis, Johannes Lemke, Sebastian Kujas, Timon Skerutsch, Urs Hoffmann, Johannes Busch, Daniel Neumayr, Julius S., Benjamin Schieck, \& Alexander Teczar), David Leonard, moovel Lab (Daniel Schmid, Markus Kreutzer, Florian Porada, Daniyar Jakupov) and moovel Group GmbH.

The authors acknowledge that this project was realized by moovel lab, which is part of moovel. When the project was realized, moovel was funded by Daimler Financial Services AG. Nonetheless the project was neither initiated nor developed in accordance to the car manufacturing unit Mercedes-Benz. Moovel Group $\mathrm{GmbH}$, which has since rebranded to REACH
NOW, focuses on providing urban mobility, a service which might in the future benefit from self-driving vehicles.

\section{References}

Aouf, R. S. (2018). Jaguar Land Rover's prototype driverless car makes eye contact with pedestrwians. Retrieved from: https://www.dezeen.com/2018/o9/o4/jaguar-land-roversprototype-driverless-car-makes-eye-contact-pedestrianstransport/

Arnall, T. (2012). Robot readable world. Retrieved from http://www. elasticspace.com/2012/o2/robot-readable-world

Awad, E., Dsouza, S., Kim, R., Schulz, J., Henrich, J., Shariff, A., Bonnefon, J., \& Rahwan, I. (2018). The Moral Machine Experiment. Nature, 563, 59-64. https://www.doi.org/10.1038/s41586-018-0637-6

Bridle, J. (2019). The New Aesthetics. Retrieved from: http://new-aesthetic.tumblr.com/

Dunne \& Raby (2013). Speculative everything: design, fiction, and social dreaming. Cambridge, MA: MIT Press.

Geuss, M (2017). "Driverless van" is just a VT researcher in a really good driver's seat costume. Retrieved from: https:// arstechnica.com/cars/2017/08/driverless-van-is-just-a-vtresearcher-in-a-really-good-drivers-seat-costume/

Giffi, et al. (2017): The race to autonomous driving: Winning American consumers' trust. Deloitte Review, 20, 73-93. Retrieved from: https://www2.deloitte.com/content/dam/ insights/us/articles/3565_Race-to-autonomous-driving/ DR20_The\%2orace\%20to\%2oautonomous\%2odriving_ reprint.pdf

IDEO (2019). The future of automobility. Retrieved from: https://automobility.ideo.com/

Jones, M. (2011). Sensor-Vernacular. Retrieved from: http://berglondon.com/blog/2011/05/13/sensor-vernacular/ KBB (2016). Future autonomous vehicle driver study. Kelly Blue Book: Cox Automotive. Retrieved from: https://mediaroom. $\mathrm{kbb} . c o m /$ download/Kelley+Blue+Book+Future+Autonomou s+Vehicle+Driver+Study+-+FINAL.pdf

Knight, W. (2017). Nvidia lets you peer inside the black box of its selfdriving AI. Retrieved from: https://www.technologyreview. $\mathrm{com} / \mathrm{s} / 604324 /$ nvidia-lets-you-peer-inside-the-black-box-ofits-self-driving-ai/ 
Sütfeld, L. R., Gast, R., König, P., \& Pipa, G. (2017). Using virtual reality to assess ethical decisions in road traffic scenarios: Applicability of value-of-life-based models and influences of time pressure. Frontiers in Behavioral Neuroscience, 11. https://doi.org/10.3389/fnbeh.2017.00122

\section{About the authors}

Joey Lee is a New York based creative technologist and geographer. His work explores relationships between the environment, data, and computation, often taking the form of experimental research, software, and visualization. Joey enjoys learning through making and uses design as a bridge to collaborate within and across disciplines.

Email: josephkanglee@gmail.com
Benedikt Groß is a speculative and computational designer, and Director of Design at moovel lab. He is fascinated by the relationships between people, their data, technology and environments. He has a MA in Design Interactions from the Royal College of Art, is co-author of "Generative Design", an acclaimed book on computation design. Benedikt is a professor of Interaction Design at the HfG Schwäbisch Gmünd.

Email: bg@benedikt-gross.de

Raphael Reimann is a multi-disciplinary urbanist. His background is found in geography and urban design and development. While playing a key role in ideation and conceptualization Raphael is mainly responsible for communication and media representation of the lab's projects. He's a founding member and perpetual advocate of moovel lab.

Email: raphael.reimann@gmail.com
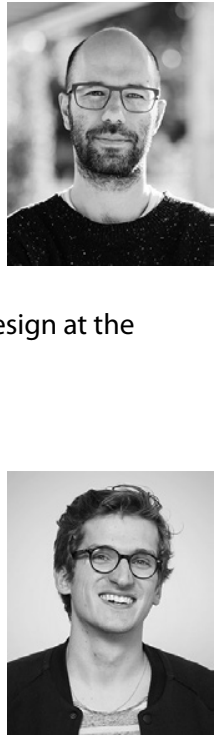